\title{
Facile Synthesis, Characterization, and In Vitro Antimicrobial Screening of a New Series of 2,4,6-Trisubstituted-s-triazine Based Compounds
}

\author{
Ravi Bhushan Singh, ${ }^{1}$ Nirupam Das, ${ }^{2}$ and Md. Kamaruz Zaman ${ }^{3}$ \\ ${ }^{1}$ Institute of Pharmacy, Harish Chandra PG College, Varanasi 221 002, India \\ ${ }^{2}$ Department of Pharmaceutical Sciences, Assam University, Silchar 788 011, India \\ ${ }^{3}$ Department of Pharmaceutical Sciences, Dibrugarh University, Dibrugarh 786 004, India \\ Correspondence should be addressed to Md. Kamaruz Zaman; zamankamaruz.du@gmail.com
}

Received 15 June 2014; Accepted 9 January 2015

Academic Editor: Arie Zask

Copyright (C) 2015 Ravi Bhushan Singh et al. This is an open access article distributed under the Creative Commons Attribution License, which permits unrestricted use, distribution, and reproduction in any medium, provided the original work is properly cited.

\begin{abstract}
A series of new 2,4,6-trisubstituted-s-triazine was synthesized, assessed for antimicrobial activity, and characterized by FTIR, ${ }^{1} \mathrm{HNMR},{ }^{13} \mathrm{CNMR}$, and elemental analysis. The tested compounds, $\mathbf{4 d}, \mathbf{4 g}, \mathbf{4 h}, \mathbf{4 k}$, and $\mathbf{4 n}$, have shown considerable in vitro antibacterial efficacy with reference to the standard drug ciprofloxacin (MIC $3.125 \mu \mathrm{gmL}^{-1}$ against B. subtilis, E. coli, and K. pneumoniae). It was observed that compounds $\mathbf{4 d}$ and $\mathbf{4 h}$ displayed equipotent antibacterial efficacy against B. subtilis (MIC $3.125 \mu \mathrm{gmL}^{-1}$ ) and S. aureus (MIC $6.25 \mu \mathrm{gmL}^{-1}$ ). The studies demonstrated that the para-fluorophenylpiperazine substituted $s$ triazine (4n) was potent and exhibited broad spectrum antibacterial activity against S. epidermidis, K. pneumoniae, and P. aeruginosa with MIC of $6.25 \mu \mathrm{gmL}^{-1}$ and for E. coli, it showed an MIC of $3.125 \mu \mathrm{gmL}^{-1}$ equipotent with reference to the standard drug. Among all the compounds under investigation, compound $\mathbf{4 g}$ also demonstrated significant antifungal activity $\left(3.125 \mu \mathrm{gmL}^{-1}\right) \mathrm{against} \mathrm{C}$. albicans.
\end{abstract}

\section{Introduction}

Over the past decades, the development of new antibiotics has plummeted while antimicrobial resistance (AMR) has increased. Only a limited number of new antimicrobials have been developed in the last decade [1] and overtreatment with the available antibiotics has led to the emergence of AMR [2]. Despite big advances in antimicrobial therapies and strategies in counteracting infections, the emergence of AMR represents an emergency situation [3]. A high percentage of hospital-acquired infections are caused by highly resistant bacteria such as methicillin-resistant Staphylococcus aureus and vancomycin-resistant enterococci [4]. Further, infections caused by resistant microorganisms often fail to respond to the conventional treatment, resulting in prolonged illness and higher death risk. Considering the scenario, the World Health Organization has necessitated an urgent and consolidated effort to avoid regressing to the preantibiotic era [5].
Therefore, discovery of novel antimicrobial molecules and their rational use are crucial to combat microbial infections. In recent years, triazine derivatives have gained particular interest on account of their broad biological activities.

Triazine based compounds are proven to possess potent anticancer [6], antiretroviral [7], antimalarial [8], and antitubercular activity [9]. Additionally, it has also been reported that certain $s$-triazine derivatives possess potent antimicrobial activity [10-12] and have been explored by researchers successfully to yield potent antimicrobial agents. For example, compounds having thiazole nucleus attached with this structural motif displayed promising antifungal activity [13]. Furthermore, we have reported certain $s$-triazine derivative substituted with diethylamino side chain and paramethoxyphenyl ring that have demonstrated broad spectrum antibacterial activity comparable to streptomycin [14]. It is noteworthy that several $s$-triazines coupled with piperazine have shown promising antimicrobial activity. In particular, 


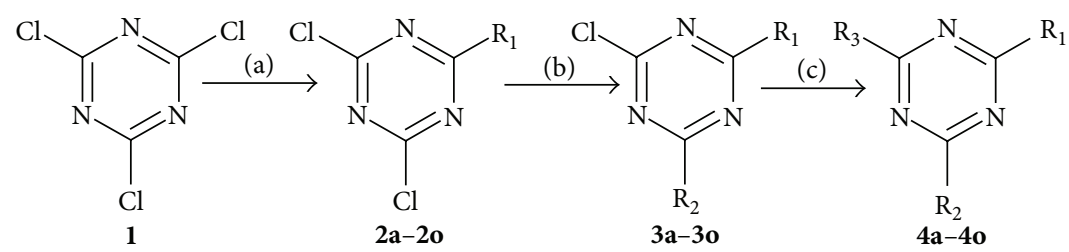

Scheme 1: General scheme for the synthesis of compounds $\mathbf{4 a}-\mathbf{4 o}$. Reagents and conditions: (a) $\mathrm{R}_{1} \mathrm{H}$, acetone, stir, $0-5^{\circ} \mathrm{C}, 2 \mathrm{~h}, \mathrm{Na}_{2} \mathrm{CO}_{3}$; (b) $\mathrm{R}_{2} \mathrm{H}$, acetone, stir, r.t., $3 \mathrm{~h}, \mathrm{Na}_{2} \mathrm{CO}_{3}$; (c) $\mathrm{R}_{3} \mathrm{H}, 1,4$ dioxane, reflux, $60-65^{\circ} \mathrm{C}, 6-8 \mathrm{~h}$.

1-(2-pyridyl) piperazine attached to the $s$-triazine nucleus containing 8-hydroxy quinoline in addition to 4-amino benzonitrile linkage showed potent activity against Escherichia coli compared to other analogues [15].

Recently, 4-benzyl piperazine derivatives of $s$-triazine were also found to be active against some Gram positive and Gram negative bacterial strains [16]. The importance of piperazine moiety has been substantiated by the fact that several $N$-alkyl and $N$-aryl piperazine derivatives showed potent antibacterial activity against resistant microbial strains $[17,18]$. Additionally, thiosemicarbazones of piperazine bearing arylmethylidene also displayed potent antimicrobial activity compared to ciprofloxacin [19]. Thus considering the above factors and with a goal to explore and improve the antimicrobial profile of $s$-triazines, we carried out the synthesis of a series of new 2,4,6-trisubstituted-s-triazines. All the compounds are sequentially substituted with aryl, diethylamino, and/or morpholinyl substituent at $2 \mathrm{nd}$, 4th, and 6 th position, respectively, of $s$-triazine. In addition, the compounds are invariably substituted with piperazine, $\mathrm{N}$ methylpiperazine or aryl substituted piperazine as one of its substituents of $s$-triazine scaffold. The precursor for the compounds is cyanuric chloride and the synthetic route involves temperature controlled nucleophilic substitution reaction. The reaction relies on differential reactivity of the substituted chlorine atoms and the easy displacement of the chloride with nucleophiles for nonsymmetrical trisubstituted functionalization [20]. The newly synthesized compounds were screened for their possible antimicrobial activity to identify new candidates as antibacterial and antifungal agents.

\section{Materials and Methods}

All the chemicals used in this experiment were of analytical grade and procured from Merck (India) and Hi-Media (India). Cyanuric chloride was obtained from Lonza Ltd., Switzerland, as a gift sample. Melting points were determined in open capillaries using Veego VMP1 melting point apparatus, Veego Instruments Corporation (Mumbai, India), and are uncorrected. The progress of the reactions was monitored by thin layer chromatography developed with nhexane/diethyl ether $(1: 1)$ and performed on Merck silica gel 60 F254 aluminium sheets and products were purified by recrystallization. IR spectra were recorded on a Perkin Elmer-Spectrum RX-1 spectrophotometer, PerkinElmer, Inc. (California, USA), on potassium bromide pellets and were recorded in $\mathrm{cm}^{-1}$. ${ }^{1} \mathrm{H} \mathrm{NMR}$ and ${ }^{13} \mathrm{C}$ NMR spectra were performed on a JEOL AL300 FT-NMR (300 MHz), Jeol Ltd., Japan, in DMSO- $\mathrm{d}_{6} / \mathrm{CDCl}_{3}$ using tetramethylsilane as internal standard and the chemical shifts are reported in ppm $(\delta)$. Elemental analysis $(\mathrm{C}, \mathrm{H}$, and $\mathrm{N})$ were performed on Exeter CE-440 elemental analyzer (Exeter Analytical Inc., USA).

2.1. Chemistry. Scheme 1 outlines the general classic threestep temperature controlled method of synthesis of 2,4,6trisubstituted-s-triazine derivatives (Table 1) as mentioned in the literature [21].

\subsection{Synthesis}

2.2.1. General Method of Synthesis of 2-Substituted-4,6dichloro-s-triazine Derivatives $(\mathbf{2 a - 2 o})$. Cyanuric chloride (1) $(0.01 \mathrm{~mol})$ was dissolved in acetone $(25 \mathrm{~mL})$ and different substituted nucleophiles $\left(\mathrm{R}_{1} \mathrm{H}\right)$ (Table 1$)(0.02 \mathrm{~mol})$ were added to it at $0-5^{\circ} \mathrm{C}$ and stirred for $2 \mathrm{~h}$. Thereafter, sodium carbonate solution (10\%) was added to neutralize the hydrochloric acid evolved during the reaction. The reaction mixture was then poured into crushed ice and the solid separated out was filtered, washed with water, and recrystallized from ethanol.

\subsubsection{General Method of Synthesis of 2,4-Disubstituted-} 6-chloro-s-triazine Derivatives (3a-3o). 2-Substituted-4,6dichloro-s-triazine derivatives $(\mathbf{2 a - 2 o})$ were added to $100 \mathrm{~mL}$ of acetone and different substituted nucleophiles $\left(\mathrm{R}_{2} \mathrm{H}\right)$ (Table 1) $(0.02 \mathrm{~mol})$ were added to it and stirred at room temperature for $3 \mathrm{~h}$. Sodium carbonate solution (10\%) was added to neutralize the hydrochloric acidevolved during the reaction and the product was filtered and washed with cold water and recrystallized from ethanol.

2.2.3. General Method of Synthesis of 2,4,6-Trisubstituteds-triazine Derivatives (4a-4o). 2,4-Disubstituted-6-chloro$s$-triazine derivatives (3a-3o) were added to $20 \mathrm{~mL}$ of 1,4-dioxane and different substituted nucleophiles $\left(\mathrm{R}_{3} \mathrm{H}\right)$ $(0.02 \mathrm{~mol})$ (Table 1$)$ were refluxed at $60-65^{\circ} \mathrm{C}$ for $6-8 \mathrm{~h}$ during which anhydrous sodium carbonate $(10 \%)$ was added in fractions $(0.2 \mathrm{~g})$ over $2 \mathrm{hr}$. The content was then poured into crushed ice; precipitate obtained was dried and recrystallized from ethanol to afford the target compounds (4a-4o). 6-(piperazin-1-yl)-s-triazine-2,4-diamine (4a). Pale brown 
TABLE 1: 2,4,6-Trisubstituted-s-triazine derivatives.

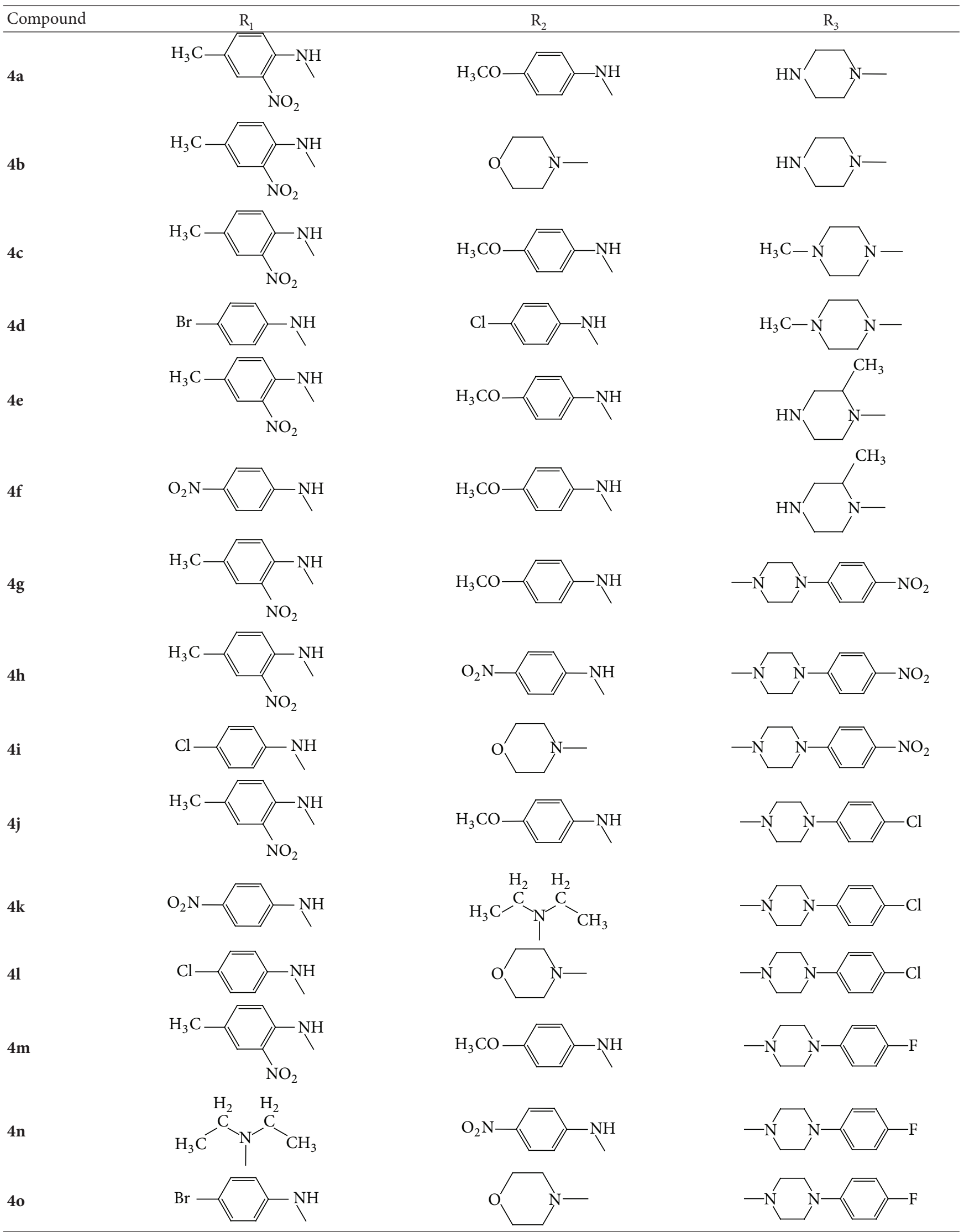


solid, Yield: $80 \%, \mathrm{mp} 155-157^{\circ} \mathrm{C}$; IR cm $\mathrm{cm}^{-1}$ (KBr): $3315\left(2^{\circ}\right.$ -NH stretch amines); 2965 (methyl $\mathrm{CH}$ asymmetric stretch); 2833 (methoxy $-\mathrm{CH}$ stretch); 1575 ( $-\mathrm{C}=\mathrm{N}$ ring stretch); 1331, 1523 (-NO stretch), 805 (CN stretch $s$-triazine). ${ }^{1} \mathrm{H}$ NMR $\left(300 \mathrm{MHz}, \mathrm{DMSO}-\mathrm{d}_{6}\right): \delta 1.98(\mathrm{~s}, 1 \mathrm{H}$, piperazinyl$\mathrm{NH}), \delta 2.56\left(\mathrm{~s}, 3 \mathrm{H},-\mathrm{CH}_{3}\right), \delta 3.21(\mathrm{t}, 4 \mathrm{H}$, piperazinyl), $\delta 3.53$ $\left(\mathrm{t}, 4 \mathrm{H}\right.$, piperazinyl), $\delta 3.78\left(\mathrm{~s}, 3 \mathrm{H},-\mathrm{OCH}_{3}\right), \delta 6.45-7.71(\mathrm{~m}$, $7 \mathrm{H}, \mathrm{ArH}), \delta 8.36$ (s, 2H, -NH). ${ }^{13} \mathrm{C}$ NMR (75 MHz, DMSO$\left.\mathrm{d}_{6}\right): \delta 181.3$ (C6 s-triazine, C-N piperazinyl linkage), 173.1 (C2 s-triazine, C-N 4-methoxyphenyl linkage), 157.5 (C4 $s$-triazine, C-N 4-methyl-2-nitrophenyl linkage), 153.3, 137.5, $134.2,129.8,117.2$ (12C aryl carbons), $56.4\left(-\mathrm{OCH}_{3}\right), 51.2,46.3$ (4C piperazinyl carbons), 23.5 ( $\mathrm{N} 4$ aryl $4-\mathrm{CH}_{3}$ ). Anal. Calcd for: $\mathrm{C}_{21} \mathrm{H}_{24} \mathrm{~N}_{8} \mathrm{O}_{3}$ : C, 57.79; H, 5.54; N, 25.67; Found: C: 57.58; $\mathrm{H}: 5.55$; N: 25.73 .

2.2.5. N-(4-Methyl-2-nitrophenyl)-4-morpholino-6-(piperazin -1-yl)-s-triazin-2-amine (4b). Yellow solid, Yield: $86 \%, \mathrm{mp}$ 160-162 ${ }^{\circ}$; IR cm ${ }^{-1}$ (KBr): 3321 ( $2^{\circ}-\mathrm{NH}$ stretch); 2966 (methyl $\mathrm{CH}$ asymmetric stretch); 1577 ( $-\mathrm{C}=\mathrm{N}$ ring str); 1334, 1527 (-NO stretch); 1117 (morpholinyl-C-O-C stretch); 809 (CN stretch $s$-triazine). ${ }^{1} \mathrm{H}$ NMR $\left(300 \mathrm{MHz}, \mathrm{CDCl}_{3}\right): \delta 1.87$ $(\mathrm{s}, 1 \mathrm{H}$, piperazinyl $-\mathrm{NH}), \delta 2.62\left(\mathrm{~s}, 3 \mathrm{H},-\mathrm{CH}_{3}\right), \delta 3.18(\mathrm{~m}$, $4 \mathrm{H}$, piperazinyl), $\delta 3.47(\mathrm{~m}, 4 \mathrm{H}$, piperazinyl), $\delta 3.65(\mathrm{t}$, $4 \mathrm{H}$, morpholinyl), $\delta 3.81$ (t, $4 \mathrm{H}$, morpholinyl), $\delta$ 6.60-7.74 $(\mathrm{m}, 3 \mathrm{H}, \mathrm{ArH}), \delta 8.47(\mathrm{~s}, 1 \mathrm{H},-\mathrm{NH}) .{ }^{13} \mathrm{C} \mathrm{NMR}(75 \mathrm{MHz}$, $\mathrm{CDCl}_{3}$ ): $\delta 183.2(\mathrm{C} 4 s$-triazine, $\mathrm{C}-\mathrm{N}$ morpholino linkage), 179.3 (C6 s-triazine, C-N piperazinyl linkage), 160.8 (C2 striazine, C-N 4-methyl-2-nitrophenyl linkage), 139.4, 136.0, 127.1, 117.2 (6C aryl carbons), 65.2, 51.7 (4C morpholino carbons), 46.7, 44.9 (4C piperazinyl carbons), 23.1 (C2 aryl 4- $\mathrm{CH}_{3}$ ). Anal. Calcd for $\mathrm{C}_{18} \mathrm{H}_{24} \mathrm{~N}_{8} \mathrm{O}_{3}$ : C, 53.99; $\mathrm{H}, 6.04 ; \mathrm{N}$, 27.98; Found: C, 53.95; H, 6.02; N, 28.01.

2.2.6. N2-(4-Methoxyphenyl)-N4-(4-methyl-2-nitrophenyl)6-(4-methylpiperazin-1-yl)-s-triazine-2,4-diamine (4c). Pale brown solid, Yield: $76 \%, \mathrm{mp} 181-183^{\circ} \mathrm{C}$; IR $\mathrm{cm}^{-1}$ (KBr): 3317 ( $2^{\circ}$-NH stretch); 2962 (methyl $\mathrm{CH}$ asymmetric stretch); 2831 (methoxy $-\mathrm{CH}$ stretch); $1570 \quad(-\mathrm{C}=\mathrm{N}$ ring stretch); 1333, 1525 (-NO stretch), 805 (CN stretch s-triazine). ${ }^{1} \mathrm{H}$ NMR $\left(300 \mathrm{MHz}, \mathrm{DMSO}-\mathrm{d}_{6}\right): \delta 2.25\left(\mathrm{~s}, 3 \mathrm{H},-\mathrm{CH}_{3}\right), \delta 2.49$ $\left(\mathrm{s}, 3 \mathrm{H},-\mathrm{CH}_{3}\right), \delta 2.98(\mathrm{t}, 4 \mathrm{H}$, piperazinyl), $\delta 3.36(\mathrm{t}, 4 \mathrm{H}$, piperazinyl), $\delta 3.75\left(\mathrm{t}, 3 \mathrm{H},-\mathrm{OCH}_{3}\right), \delta 6.37-7.78(\mathrm{~m}, 7 \mathrm{H}$, ArH), $\delta 8.63(\mathrm{~s}, 2 \mathrm{H}, \mathrm{NH}),{ }^{13} \mathrm{C}$ NMR (75 MHz, DMSO$\left.\mathrm{d}_{6}\right): \delta 180.6$ (C6 $s$-triazine, C-N piperazinyl linkage), 164.8 (C4 s-triazine, C-N 4-methyl-2-nitrophenyl linkage), 158.9 (C2 s-triazine, C-N 4-methoxyphenyl linkage), 138.5, 136.1, 134.5, 130.3, 124.9 117.1, 114.1 (12C aryl carbons), 56.7 (N2 aryl $4-\mathrm{OCH}_{3}$ ), 52.2, 47.7 (4C piperazinyl carbons), 42.5 (C6 piperazinyl $\left.4-\mathrm{CH}_{3}\right), 23.4\left(\mathrm{~N} 4\right.$ aryl $\left.4-\mathrm{CH}_{3}\right)$. Anal. Calcd for: $\mathrm{C}_{22} \mathrm{H}_{26} \mathrm{~N}_{8} \mathrm{O}_{3}$ : C, 58.65; H, 5.82; N, 24.87; Found: C: 57.48; H: 5.83; N: 24.85 .

2.2.7. N2-(4-Bromophenyl)-N4-(4-chlorophenyl)-6-(4-methylpiperazin-1-yl)-s-triazine-2,4-diamine (4d). Pale brown solid, Yield: $78 \%, \mathrm{mp} 171-173^{\circ} \mathrm{C}$; IR $\mathrm{cm}^{-1}(\mathrm{KBr})$ : $3321\left(2^{\circ}-\mathrm{NH}\right.$ stretch); 1575 ( $-\mathrm{C}=\mathrm{N}$ ring stretch); 810 (CN stretch $s$-triazine).
${ }^{1} \mathrm{H}$ NMR (300 MHz, DMSO-d $\left.\mathrm{d}_{6}\right): \delta 2.25\left(\mathrm{~s}, 3 \mathrm{H},-\mathrm{CH}_{3}\right), \delta 3.13$ (t, $4 \mathrm{H}$, piperazinyl), 3.31 (t, $4 \mathrm{H}$, piperazinyl), $\delta 6.35-7.74(\mathrm{~m}$, $8 \mathrm{H}, \mathrm{ArH}), \delta 8.54$ (s, 2H, NH). ${ }^{13} \mathrm{C}$ NMR (75 MHz, DMSO$\left.\mathrm{d}_{6}\right): \delta 181.2$ (C6 s-triazine, C-N piperazinyl linkage), 166.4 (C2 s-triazine, C-N 4-bromophenyl linkage), 159.2 (C4striazine, C-N 4-chlorophenyl linkage), 139.3, 136.0, 135.4, 133.7, 130.3, $126.1118 .5,116.9$ (12C aryl carbons), 54.7, 45.8 (4C piperazinyl carbons), 43.1 (C6 piperazinyl 4- $\mathrm{CH}_{3}$ ). Anal. Calcd for: $\mathrm{C}_{20} \mathrm{H}_{21} \mathrm{BrClN}_{7}$ : C, 50.59; H, 4.46; N, 20.65 Found: C: $50.44 ; \mathrm{H}: 4.45 ; \mathrm{N}: 20.60$.

2.2.8. N2-(4-Methoxyphenyl)-N4-(4-methyl-2-nitrophenyl)6-(2-methylpiperazin-1-yl)-s-triazine-2,4-diamine (4e). Pale brown solid, Yield: $82 \%$, mp 210-212 ${ }^{\circ}$; IR cm ${ }^{-1}$ (KBr): 3315 $\left(2^{\circ}-\mathrm{NH}\right.$ stretch); 2965 (methyl $\mathrm{CH}$ asymmetric stretch); 2835 (methoxy $-\mathrm{CH}$ stretch); 1575 ( $-\mathrm{C}=\mathrm{N}$ ring stretch); 1334, 1530 (-N-O stretch); 805 (CN stretch $s$-triazine). ${ }^{1} \mathrm{H}$ NMR $\left(300 \mathrm{MHz}, \mathrm{DMSO}-\mathrm{d}_{6}\right): \delta 2.08(\mathrm{~s}, 1 \mathrm{H},-\mathrm{NH}), \delta 2.20$ $\left(\mathrm{s}, 3 \mathrm{H},-\mathrm{CH}_{3}\right), \delta 2.55\left(\mathrm{~s}, 3 \mathrm{H},-\mathrm{CH}_{3}\right), \delta 2.79-3.23(\mathrm{~m}, 7 \mathrm{H}$, piperazinyl), $\delta 3.73\left(\mathrm{~s}, 3 \mathrm{H},-\mathrm{OCH}_{3}\right), \delta 6.56-7.81(\mathrm{~m}, 7 \mathrm{H}$, $\mathrm{Ar}-\mathrm{H}), \delta 8.37$ (s, 2H, NH). ${ }^{13} \mathrm{C}$ NMR (75 MHz, DMSO$\left.\mathrm{d}_{6}\right): \delta 181.2$ (C6 s-triazine, C-N piperazinyl linkage), 166.7 (C4 s-triazine, C-N 4-methyl-2-nitrophenyl linkage), 160.3 (C4 s-triazine, C-N 4-methoxyphenyl linkage), 138.5, 136.6, $134.8,135.2,130.3,124.9,117.1,115.1$ (12C aryl carbons), 59.5, 57.6 (piperazinyl), $55.9\left(\mathrm{~N} 2\right.$ aryl $\left.4-\mathrm{OCH}_{3}\right), 50.4$, 47.1 (piperazinyl), 23.4 ( $\mathrm{N} 4$ aryl $4-\mathrm{CH}_{3}$ ), 16.5 (C6 piperazinyl 2- $\mathrm{CH}_{3}$ ). Anal. Calcd for: $\mathrm{C}_{22} \mathrm{H}_{26} \mathrm{~N}_{8} \mathrm{O}_{3}: \mathrm{C}, 58.65 ; \mathrm{H}, 5.82 ; \mathrm{N}, 24.87$; Found: C: 58.63 ; H: 5.84; N: 24.85.

2.2.9. N2-(4-Methoxyphenyl)-6-(2-methylpiperazin-1-yl)-N4(4-nitrophenyl)-s-triazine-2,4-diamine (4f). Brown solid, Yield: $82 \%, \mathrm{mp} 207-209^{\circ} \mathrm{C}$; IR $\mathrm{cm}^{-1}$ (KBr): $3318\left(2^{\circ}-\mathrm{NH}\right.$ stretch); 2830 (methoxy $-\mathrm{CH}$ stretch); 1570 (-C=N ring stretch); 1333, 1528 (NO stretch); 808 (CN stretch $s$-triazine). ${ }^{1} \mathrm{H}$ NMR (300 MHz, DMSO-d 6 ): $\delta 1.98(\mathrm{~s}, 1 \mathrm{H},-\mathrm{NH}), \delta 2.21$ $\left(\mathrm{s}, 3 \mathrm{H},-\mathrm{CH}_{3}\right), \delta 2.75-3.31(\mathrm{~m}, 7 \mathrm{H}$, piperazinyl), $\delta 3.74(\mathrm{~s}$, $3 \mathrm{H},-\mathrm{OCH}_{3}$ ), $\delta$ 6.36-7.93 (s, 8H, ArH), $\delta 8.33$ (s, 2H, NH). ${ }^{13} \mathrm{C}$ NMR (75 MHz, DMSO-d 6 ): $\delta 180.1$ (C6 s-triazine, C-N piperazinyl linkage), 165.5 (C4s-triazine, $\mathrm{C}-\mathrm{N} 4$-nitrophenyl linkage), 158.1 (C2 s-triazine, C-N 4-methoxyphenyl linkage), 149.2, 138.4, 134.6, 129.5, 121.8, 117.6 (12C aryl carbons), 61.4 (piperazinyl), 56.5 ( $\mathrm{N} 2$ aryl $\left.4-\mathrm{OCH}_{3}\right), 51.7$, 49.3, 46.2 (piperazinyl), 16.7 (C6 piperazinyl 2- $\mathrm{CH}_{3}$ ). Anal. Calcd for: $\mathrm{C}_{21} \mathrm{H}_{24} \mathrm{~N}_{8} \mathrm{O}_{3}$ : C, 57.79; H, 5.54; N, 25.67; Found: C: 57.92 ; H: 5.53; N: 25.59 .

2.2.10. N2-(4-Methoxyphenyl)-N4-(4-methyl-2-nitrophenyl)-6 -(4-(4-nitrophenyl)piperazin-1-yl)-s-triazine-2,4-diamine (4g). Pale yellow solid, Yield: $84 \%, \mathrm{mp} 190-192^{\circ} \mathrm{C}$; IR $\mathrm{cm}^{-1}$ (KBr): 3324 ( $2^{\circ}-\mathrm{NH}$ stretch); 2963 (methyl $\mathrm{CH}$ asymmetric stretch); 2837 (methoxy $-\mathrm{CH}$ stretch); 1577 ( $-\mathrm{C}=\mathrm{N}$ ring stretch); 1335, 1531 (NO stretch); 809 (CN stretch $s$-triazine). ${ }^{1} \mathrm{H}$ NMR $\left(300 \mathrm{MHz}, \mathrm{DMSO}-\mathrm{d}_{6}\right): \delta 2.37\left(\mathrm{~s}, 3 \mathrm{H},-\mathrm{CH}_{3}\right), \delta 3.22$ $(\mathrm{t}, 4 \mathrm{H}$, piperazinyl), $\delta 3.61(\mathrm{t}, 4 \mathrm{H}$, piperazinyl), $\delta 3.75(\mathrm{~s}$, $\left.3 \mathrm{H},-\mathrm{OCH}_{3}\right), \delta 6.38-8.01(\mathrm{~s}, 11 \mathrm{H}, \mathrm{ArH}), \delta 8.36(\mathrm{~s}, 2 \mathrm{H}$, 
-NH). ${ }^{13} \mathrm{C}$ NMR $(75 \mathrm{MHz}$, DMSO-d 6 ): $\delta 179.8$ (C6 striazine, $\mathrm{C}-\mathrm{N}$ piperazinyl linkage), 164.6 (C4 s-triazine, $\mathrm{C}-$ $\mathrm{N}$ 4-methyl-2-nitrophenyl linkage), 160.7 (C2 s-triazine, CN 4-methoxyphenyl linkage), 156.5, 151.3, 137.8, 136.1, 134.9, $130.3,124.6,119.3,116.2$ (18C aryl carbons), 56.3 (N2 aryl 4 $-\mathrm{OCH}_{3}$ ), 50.9, 46.4 (4C piperazinyl carbons), 23.2 (N4 aryl 4- $\mathrm{CH}_{3}$ ). Anal. Calcd for: $\mathrm{C}_{27} \mathrm{H}_{27} \mathrm{~N}_{9} \mathrm{O}_{5}$ : C, 58.16; $\mathrm{H}, 4.88 ; \mathrm{N}$, 22.61; Found: C: 58.21; H: 4.87; N: 22.67.

2.2.11. N2-(4-Methyl-2-nitrophenyl)-N4-(4-nitrophenyl)-6-(4(4-nitrophenyl)piperazin-1-yl)-s-triazine-2,4-diamine (4h). Pale yellow solid, Yield: $84 \%$, mp $213-215^{\circ} \mathrm{C}$; IR $\mathrm{cm}^{-1}(\mathrm{KBr})$ : 3319 ( $2^{\circ}-\mathrm{NH}$ stretch); 2963 (methyl $\mathrm{CH}$ asymmetric stretch); 1575 (-C=N ring stretch); 1331, 1528 (NO stretch); 809 (CN stretch $s$-triazine). ${ }^{1} \mathrm{H}$ NMR $\left(75 \mathrm{MHz}, \mathrm{DMSO}-\mathrm{d}_{6}\right): \delta 2.37$ $\left(\mathrm{s}, 3 \mathrm{H},-\mathrm{CH}_{3}\right), \delta 3.24(\mathrm{t}, 4 \mathrm{H}$, piperazinyl), $\delta 3.45(\mathrm{t}, 4 \mathrm{H}$, piperazinyl), $\delta 6.65-8.18(\mathrm{~m}, 11 \mathrm{H}, \mathrm{ArH}), \delta 8.31(\mathrm{~s}, 2 \mathrm{H},-\mathrm{NH})$. ${ }^{13} \mathrm{C}$ NMR $\left(75 \mathrm{MHz}, \mathrm{DMSO}-\mathrm{d}_{6}\right.$ ): $\delta 180.6$ (C6 $s$-triazine, C-N piperazinyl linkage), 167.9 (C2 s-triazine, C-N 4-methyl-2nitrophenyl linkage), 159.2 (C4s-triazine, $\mathrm{C}-\mathrm{N} 4$-nitrophenyl linkage), 156.4 148.8, 136.5, 127.6, 122.0, 118.9 116.2 (18C aryl carbons), 49.6, 46.7 (4C piperazinyl carbons), 23.5 (N2 aryl 4- $\mathrm{CH}_{3}$ ). Anal. Calcd for: $\mathrm{C}_{26} \mathrm{H}_{24} \mathrm{~N}_{10} \mathrm{O}_{6}: \mathrm{C}, 54.54 ; \mathrm{H}, 4.23 ; \mathrm{N}$, 24.46; Found: C: 54.73; H: 4.22; N: 24.51.

2.2.12. N-(4-Chlorophenyl)-4-morpholino-6-(4-(4-nitrophenyl) piperazin-1-yl)-s-triazine-2-amine (4i). Pale yellow solid, Yield: $84 \%, \mathrm{mp} 165-167^{\circ} \mathrm{C}$; IR $\mathrm{cm}^{-1}(\mathrm{KBr}): 3323\left(2^{\circ}-\mathrm{NH}\right.$ stretch); 1573 ( $-\mathrm{C}=\mathrm{N}$ ring stretch); 1119 (morpholinyl CO-C stretch); 1336, 1527 (NO stretch); 810 (CN stretch $s$-triazine). ${ }^{1} \mathrm{H}$ NMR $\left(300 \mathrm{MHz}, \quad\right.$ DMSO- $\left.\mathrm{d}_{6}\right): \delta 3.21 \quad(\mathrm{t}$, $4 \mathrm{H}$, piperazinyl), $\delta 3.41$ (t, $4 \mathrm{H}$, piperazinyl), $\delta 3.56(\mathrm{t}$, $4 \mathrm{H}$, morpholino), $\delta 3.84(\mathrm{t}, 4 \mathrm{H}$, morpholino), $\delta$ 6.55-8.16 $(\mathrm{m}, 8 \mathrm{H}, \mathrm{ArH}), \delta 8.48(\mathrm{~s}, 1 \mathrm{H},-\mathrm{NH}) .{ }^{13} \mathrm{C} \mathrm{NMR}(75 \mathrm{MHz}$, DMSO- $\mathrm{d}_{6}$ ): $\delta 182.4$ (C6 $s$-triazine, $\mathrm{C}-\mathrm{N}$ piperazinyl linkage), 179.7 (C4 s-triazine, C-N morpholino linkage), 165.2 (C2 $s$-triazine, C-N 4-chlorophenyl linkage), 151.3, 146.2, 130.8, 126.5, 119.1, 115.2 (12C aryl carbons), 66.4 (morpholino), 50.7, 48.3 (4C piperazinyl carbons), 46.5 (morpholino). Anal. Calcd for: $\mathrm{C}_{23} \mathrm{H}_{25} \mathrm{ClN}_{8} \mathrm{O}_{3}$ : C, 55.59; H, 5.07; N, 22.55; Found: C: $55.61 ; \mathrm{H}: 5.18 ; \mathrm{N}: 22.48$.

2.2.13. 6-(4-(4-Chlorophenyl)piperazin-1-yl)-N2-(4-methoxyphenyl)-N4-(4-methyl-2-nitrophenyl)-s-triazine-2,4-diamine (4j). Yellow solid, Yield: $84 \%, \mathrm{mp} 208-210^{\circ} \mathrm{C}$; IR cm ${ }^{-1}(\mathrm{KBr})$ : 3321 ( $2^{\circ}-\mathrm{NH}$ stretch); 2835 (methoxy -CH stretch); 2964 (methyl $\mathrm{CH}$ asymmetric stretch); 1577 ( $-\mathrm{C}=\mathrm{N}$ ring stretch); 1336, 1528 (NO stretch); 809 (CN stretch $s$-triazine). ${ }^{1} \mathrm{H}$ NMR $\left(300 \mathrm{MHz}, \mathrm{DMSO}-\mathrm{d}_{6}\right): \delta 2.42\left(\mathrm{~s}, 3 \mathrm{H},-\mathrm{CH}_{3}\right), \delta 3.18$ $(\mathrm{t}, 4 \mathrm{H}$, piperazinyl), $\delta 3.51(\mathrm{t}, 4 \mathrm{H}$, piperazinyl), $\delta 3.73$ $\left(\mathrm{s}, 3 \mathrm{H},-\mathrm{OCH}_{3}\right), \delta 6.35-7.87(\mathrm{~m}, 11 \mathrm{H}, \operatorname{ArH}), \delta 8.43(\mathrm{~s}$, $2 \mathrm{H},-\mathrm{NH}) .{ }^{13} \mathrm{C}$ NMR $\left(75 \mathrm{MHz}, \mathrm{DMSO}_{-} \mathrm{d}_{6}\right): \delta 180.8$ (C6 $s$-triazine, $\mathrm{C}-\mathrm{N}$ piperazinyl linkage), 165.6 (C4 s-triazine, C-N 4-methyl-2-nitrophenyl linkage), 163.5 (C2 s-triazine, C-N 4-methoxyphenyl linkage), 155.4, 138.0, 135.6, 134.9, 128.7, 117.2, 114.7 (18C aryl carbons), 56.9 ( $\mathrm{N} 2$ aryl $\left.4-\mathrm{OCH}_{3}\right)$, 49.6, 46.2 (4C piperazinyl carbons), 23.5 ( $\mathrm{N} 4$ aryl $4-\mathrm{CH}_{3}$ ).
Anal. Calcd for: $\mathrm{C}_{27} \mathrm{H}_{27} \mathrm{ClN}_{8} \mathrm{O}_{3}$ : C, 59.28; H, 4.98; N, 20.48; Found: C: 59.19; H: 4.97; N: 20.61.

2.2.14. 6-(4-(4-Chlorophenyl)piperazin-1-yl)-N2,N2-diethylN4-(4-nitrophenyl)-s-triazine-2,4-diamine (4k). Yellow solid, Yield: $84 \%, \mathrm{mp} 211-213^{\circ} \mathrm{C}$; IR $\mathrm{cm}^{-1}$ (KBr): $3316\left(2^{\circ}-\right.$ $\mathrm{NH}$ stretch); $1570(-\mathrm{C}=\mathrm{N}$ ring stretch); 1337, 1528 (NO stretch); 1314 ( $3^{\circ} \mathrm{CN}$ stretch); 805 (CN stretch $s$-triazine). ${ }^{1} \mathrm{H}$ NMR $\left(300 \mathrm{MHz}, \mathrm{DMSO}-\mathrm{d}_{6}\right): \delta 2.23\left(\mathrm{~s}, 4 \mathrm{H},-\mathrm{CH}_{2}\right), \delta 3.18$ $\left(\mathrm{t}, 4 \mathrm{H}\right.$, piperazinyl), $\delta 3.33\left(\mathrm{~s}, 6 \mathrm{H},-\mathrm{CH}_{3}\right), 3.54(\mathrm{t}, 4 \mathrm{H}$, piperazinyl), $\delta$ 6.49-8.05 (s, 8H, ArH), $\delta 8.52(\mathrm{~s}, 1 \mathrm{H},-\mathrm{NH})$. ${ }^{13} \mathrm{C}$ NMR $\left(75 \mathrm{MHz}, \mathrm{DMSO}-\mathrm{d}_{6}\right): \delta 181.5$ (C6 s-triazine, C-N piperazinyl linkage), 179.7 (C2 $s$-triazine, $\mathrm{C}-\mathrm{N}$ diethylamino linkage), 165.4 (C4 s-triazine, $\mathrm{C}-\mathrm{N}$ 4-nitrophenyl linkage), 151.8, 147.7, 145.2, 136.3, 130.8, 117.5, 115.2 (12C aryl carbons), 51.9, 49.4 (4C piperazinyl carbons), 44.6 (4C diethyl $-\mathrm{CH}_{2}$ ), 18.3 (6C diethyl $-\mathrm{CH}_{3}$ ). Anal. Calcd for: $\mathrm{C}_{23} \mathrm{H}_{27} \mathrm{ClN}_{8} \mathrm{O}_{2}: \mathrm{C}$, 57.20; H, 5.63; N, 23.20; Found: C: 57.36; H: 5.61; N: 23.25.

2.2.15. N-(4-Chlorophenyl)-4-(4-(4-chlorophenyl)piperazin-1yl)-6-morpholino-s-triazin-2-amine (4l). Pale yellow solid, Yield: $85 \%, \mathrm{mp} 205-207^{\circ} \mathrm{C}$; IR $\mathrm{cm}^{-1}$ (KBr): $3324\left(2^{\circ}-\right.$ $\mathrm{NH}$ stretch); 1576 ( $-\mathrm{C}=\mathrm{N}$ ring stretch); 1118 (morpholinyl $\mathrm{C}-\mathrm{O}-\mathrm{C}$ stretch); 807 (CN stretch $s$-triazine). ${ }^{1} \mathrm{H}$ NMR $\left(300 \mathrm{MHz}, \mathrm{DMSO}-\mathrm{d}_{6}\right): \delta 3.19(\mathrm{t}, 4 \mathrm{H}$, piperazinyl), $\delta 3.44(\mathrm{t}$, $4 \mathrm{H}$, piperazinyl), $\delta 3.53$ (t, $4 \mathrm{H}$, morpholinyl), $\delta 3.80(\mathrm{t}, 4 \mathrm{H}$, morpholinyl), $\delta$ 6.65-7.72 (m, 8H, Ar-H), $8.43 \delta(\mathrm{s}, 1 \mathrm{H}$, $\mathrm{NH}) .{ }^{13} \mathrm{C}$ NMR $\left(75 \mathrm{MHz}, \mathrm{DMSO}-\mathrm{d}_{6}\right): \delta 183.1$ (C4s-triazine, $\mathrm{C}-\mathrm{N}$ piperazinyl linkage), 178.3 (C6 $s$-triazine, C-N morpholino linkage), 167.6 (C2 s-triazine, C-N 4-chlorophenyl linkage), 148.2, 144.5, 130.5, 124.3, 118.2, 116.1 (12C aryl carbons), 65.3 (morpholino), 51.4, 49.2 (4C piperazinyl carbons), 46.9 (morpholino). Anal. Calcd for: $\mathrm{C}_{23} \mathrm{H}_{25} \mathrm{Cl}_{2} \mathrm{~N}_{7} \mathrm{O}$ : C, 56.79; H, 5.18; N, 20.16; Found: C: 56.62; H: 5.20; N: 20.23.

2.2.16. 6-(4-(4-Fluorophenyl)piperazin-1-yl)-N2-(4-methoxyphenyl)-N4-(4-methyl-2-nitrophenyl)-s-triazine-2,4-diamine (4m). Yellow solid, Yield: $84 \%, \mathrm{mp} 184-186^{\circ} \mathrm{C}$; IR $\mathrm{cm}^{-1}$ (KBr): 3321 ( $2^{\circ}-\mathrm{NH}$ stretch); 2961 (methyl $\mathrm{CH}$ asymmetric stretch); 2830 (methoxy $\mathrm{CH}$ stretch); 1575 (-C=N ring stretch); 809 (CN stretch $s$-triazine). ${ }^{1} \mathrm{H}$ NMR $(300 \mathrm{MHz}$, DMSO- $\left.\mathrm{d}_{6}\right): \delta 2.49\left(\mathrm{~s}, 3 \mathrm{H},-\mathrm{CH}_{3}\right), \delta 3.16(\mathrm{t}, 4 \mathrm{H}$, piperazinyl), $\delta 3.55\left(\mathrm{t}, 4 \mathrm{H}\right.$, piperazinyl), $\delta 3.71\left(\mathrm{~s}, 3 \mathrm{H},-\mathrm{OCH}_{3}\right)$, $\delta$ 6.39-7.96 (m, 11H, ArH), $\delta 8.41(\mathrm{~s}, 2 \mathrm{H}, \mathrm{NH}) .{ }^{13} \mathrm{C} \mathrm{NMR}$ $\left(75 \mathrm{MHz}, \mathrm{DMSO}-\mathrm{d}_{6}\right): \delta 181.5$ (C6 $s$-triazine, $\mathrm{C}-\mathrm{N}$ piperazinyl linkage), 166.8 (C4 s-triazine, $\mathrm{C}-\mathrm{N}$ 4-methyl-2-nitrophenyl linkage), 162.2 (C2 s-triazine, C-N 4-methoxyphenyl linkage), 152.4, 137.3, 135.1, 133.4, 129.2, 116.3, 113.9 (18C aryl carbons), 56.4 (N2 aryl $\left.4-\mathrm{OCH}_{3}\right), 49.6,48.5(4 \mathrm{C}$ piperazinyl carbons), 23.6 ( $\mathrm{N} 4$ aryl $\left.4-\mathrm{CH}_{3}\right)$. Anal. Calcd for: $\mathrm{C}_{27} \mathrm{H}_{27} \mathrm{FN}_{8} \mathrm{O}_{3}$ : C, 61.12; H, 5.13; N, 21.12; Found: C: 61.22; $\mathrm{H}$ : 5.14; N: 21.19.

2.2.17. N2,N2-Diethyl-6-(4-(4-fluorophenyl)piperazin-1-yl)N4-(4-nitrophenyl)-s-triazine-2,4-diamine (4n). Pale yellow solid, Yield: $80 \%, \mathrm{mp} 202-204^{\circ} \mathrm{C}$; IR $\mathrm{cm}^{-1}$ (KBr): $3323\left(2^{\circ}-\right.$ $\mathrm{NH}$ stretch); 1577 (-C=N ring stretch); $1312\left(3^{\circ}-\mathrm{CN}\right.$ stretch); 
1335, 1526 (-NO stretch); 805 (CN stretch s-triazine). ${ }^{1} \mathrm{H}$ NMR $\left(300 \mathrm{MHz}, \mathrm{DMSO}-\mathrm{d}_{6}\right): \delta 2.17\left(\mathrm{~s}, 6 \mathrm{H},-\mathrm{CH}_{3}\right), \delta 3.21$ $\left(\mathrm{t}, 4 \mathrm{H}\right.$, piperazinyl), $\delta 3.26\left(\mathrm{~s}, 4 \mathrm{H},-\mathrm{CH}_{2}\right), \delta 3.52(\mathrm{t}, 4 \mathrm{H}$, piperazinyl), $\delta$ 6.56-8.13 (m, $8 \mathrm{H}, \mathrm{ArH}), \delta 8.45(\mathrm{~s}, 1 \mathrm{H}, \mathrm{NH})$. ${ }^{13} \mathrm{C}$ NMR (75 MHz, DMSO- $\mathrm{d}_{6}$ ): $\delta 180.3$ (C6 $s$-triazine, C-N piperazinyl linkage), 179.1 (C2 $s$-triazine, $\mathrm{C}-\mathrm{N}$ diethylamino linkage), 164.8 (C4 s-triazine, C-N 4-nitrophenyl linkage), $150.7,148.6,144.1,137.4,129.7,117.7,114.9$ (12C aryl carbons), 50.4, 48.7 (4C piperazinyl carbons), 43.2 (4C diethyl $-\mathrm{CH}_{2}$ ), 18.3 (6C diethyl $-\mathrm{CH}_{3}$ ). Anal. Calcd for: $\mathrm{C}_{23} \mathrm{H}_{27} \mathrm{FN}_{8} \mathrm{O}_{2}: \mathrm{C}$, 59.22; H, 5.83; N, 24.02; Found: C: 59.40; H: 5.85; N: 20.07.

2.2.18. N-(4-Bromophenyl)-4-(4-(4-fluorophenyl)piperazin1-yl)-6-morpholino-s-triazin-2-amine (4o). Pale yellow solid, Yield: $80 \%$, mp $194-196^{\circ} \mathrm{C}$; IR $\mathrm{cm}^{-1}$ (KBr): 3317 ( $2^{\circ}-\mathrm{NH}$ stretch); $1573 \quad(-\mathrm{C}=\mathrm{N}$ ring stretch); 1121 (morpholinyl C-O-C), 810 (CN stretch $s$-triazine). ${ }^{1} \mathrm{H}$ NMR $\left(75 \mathrm{MHz}, \mathrm{DMSO}_{-} \mathrm{d}_{6}\right): \delta 3.23(\mathrm{~m}, 4 \mathrm{H}$, piperazinyl), $\delta 3.46$ (m, $4 \mathrm{H}$, piperazinyl), $\delta 3.51$ (t, $4 \mathrm{H}$, morpholinyl), $\delta 3.83$ (t, 4H, morpholinyl), $\delta 6.39-7.44(\mathrm{~m}, 8 \mathrm{H}, \operatorname{ArH}), \delta 8.42$ $(\mathrm{s}, 1 \mathrm{H}, \mathrm{NH}) .{ }^{13} \mathrm{C}$ NMR $\left(300 \mathrm{MHz}, \mathrm{DMSO}_{-} \mathrm{d}_{6}\right): \delta 181.6(\mathrm{C} 4$ $s$-triazine, C-N piperazinyl linkage), 179.3 (C6 s-triazine, C-N morpholino linkage), 158.7 (C2 s-triazine, C-N 4bromophenyl linkage), 153.2, 143.4, 131.5, 119.5, 117.6, 115.9 (12C aryl carbons), 65.6 (morpholino), 50.2, 49.8 (4C piperazinyl carbons), 46.5 (mopholino). Anal. Calcd for: $\mathrm{C}_{23} \mathrm{H}_{25} \mathrm{BrFN}_{7} \mathrm{O}$ : C, 53.70; H, 4.90; N, 19.06; Found: C: 53.50; $\mathrm{H}: 4.88 ; \mathrm{N}: 19.11$.

2.3. In Vitro Antimicrobial Activity. The antimicrobial potency of all the synthesized compounds was assessed in vitro in accordance with the guidelines of Clinical Laboratory and Standards Institute [22] against four representative Gram positive bacteria, namely, Bacillus subtilis (ATCC 6633, NCIM 2063), Bacillus cereus (ATCC 10876, NCIM 2156), Staphylococcus epidermidis (ATCC 12228, NCIM 2493), and Staphylococcus aureus (NCIM 2079); Gram negative bacteria, namely, Escherichia coli (ATCC 8739, NCIM 2065), Klebsiella pneumoniae (NCIM 2706), Proteus mirabilis (NCIM 2241), and Pseudomonas aeruginosa (ATCC 19429, NCIM 2036); and two fungal strains Candida albicans (ATCC 2091, NCIM 3102) and Candida tropicalis (ATCC 13803, NCIM 3556). The microbial strains employed for the activity were procured from National Collection of Industrial Microorganisms (NCIM), Pune, India. Ciprofloxacin was used as the standard antibacterial drug and fluconazole as the standard antifungal drug.

2.4. Preparation of Seed Organisms. $13.0 \mathrm{~g}$ of nutrient broth medium was suspended in $1000 \mathrm{~mL}$ distilled water. Then it was boiled to dissolve the medium completely and filtered from $5 \mu$ sintered glass filter. A set of test tubes with nutrient broth $(5 \mathrm{~mL})$ was capped with cotton plugs and sterilized by autoclaving at 15 psig pressure $\left(121^{\circ} \mathrm{C}\right)$ for $15 \mathrm{~min}$. Then after cooling, a loop of organism was inoculated into liquid broth and incubated at $37 \pm 1^{\circ} \mathrm{C}$ and used within $12 \mathrm{~h}$. The inoculum size for test strain was adjusted to $10^{8} \mathrm{CFU} / \mathrm{mL}$ (colony forming unit per milliliter) by comparing the turbidity.
2.5. Determination of Zone of Inhibition (Disc Diffusion Method). Petri plates containing $15 \mathrm{~mL}$ of Mueller Hinton agar media (Hi-Media) were used for all the bacterial strains and Sabouraud dextrose agar (SDA) media (Hi-Media) were used for the fungal strains. $0.1 \mathrm{~mL}$ of seed of various organisms was aseptically inoculated over the sterile solid agar medium. Whatman no. 1 filter paper discs (6 $\mathrm{mm}$ in diameter) impregnated with the synthesized compound $(10 \mu \mathrm{g} / \mathrm{disc})$ dissolved in dimethyl sulfoxide (DMSO) were placed on the plates. All the sensitivity plates were then incubated at $37 \pm 1^{\circ} \mathrm{C}$ for $24 \mathrm{~h}$ for bacterial strain and $72 \mathrm{~h}$ at $25^{\circ} \mathrm{C}$ for fungal strain. Ciprofloxacin $(10 \mu \mathrm{g} / \mathrm{disc}, \mathrm{Hi}-\mathrm{Media})$ and fluconazole $(10 \mu \mathrm{g} / \mathrm{disc}$, Hi-Media) were used as positive control for the assessment of antibacterial and antifungal activity, respectively. A paper disc impregnated with DMSO was used as negative control. The zone of inhibition on agar plate was measured in millimeters $(\mathrm{mm})$ and the test was performed in triplicate and the average was taken as final reading (Table 2 ).

2.6. Determination of Minimum Inhibitory Concentration. Compounds that displayed favorable zone of inhibition were considered for further assessment of minimum inhibitory concentration (MIC). MIC of a compound is defined as the lowest concentration at which it completely inhibits visible growth (turbidity on liquid media). Solutions of the test compounds, ciprofloxacin and fluconazole, were prepared in DMSO at a concentration of $100 \mu \mathrm{g} / \mathrm{mL}$. From this stock solution, serial broth dilutions of the compounds $(3.125,6.25$, $12.5,25,50$, and $100 \mu \mathrm{g} / \mathrm{mL}$ ) were prepared to determine the MIC. All the determinations were done in triplicate and the average was taken as the final reading. The standard antibiotics, ciprofloxacin and fluconazole $(100 \mu \mathrm{g} / \mathrm{mL})$, were used as positive controls and $100 \mu \mathrm{g} / \mathrm{mL}$ of DMSO was used as a negative control. At the end of the incubation period $\left(37 \pm 1^{\circ} \mathrm{C}\right.$ for $\left.24 \mathrm{~h}\right)$, the MIC values were determined (Table 3$)$.

\section{Results and Discussion}

3.1. Chemistry. Synthesis of the intermediates and target compounds was accomplished according to the steps illustrated in Scheme 1. The structure of the synthesized compounds was confirmed on the basis of IR, ${ }^{1} \mathrm{H} \mathrm{NMR},{ }^{13} \mathrm{C}$ $\mathrm{NMR}$, and elemental analysis and the spectral data are in accordance with the structures of the compounds (4a-4o). Only significant bands from IR are reported. The derivatives showed characteristic band in the range of $1570-1577 \mathrm{~cm}^{-1}$ for $-\mathrm{C}=\mathrm{N}$ stretching of triazine nucleus. Further, strong absorption band in the range of $805-810 \mathrm{~cm}^{-1}$ indicates $\mathrm{CN}$ stretch of $s$-triazine moiety. A characteristic band appearing in the range of $1117-1121 \mathrm{~cm}^{-1}$ corresponds to the $\mathrm{C}-\mathrm{O}-\mathrm{C}$ stretching of morpholine in the IR spectra of compounds 4b, 4i, and 4o. The compounds also displayed $-\mathrm{NH}$ stretch for secondary amines that appears in the region 3315$3324 \mathrm{~cm}^{-1}$. The ${ }^{1} \mathrm{H}$ NMR spectra of all the derivatives $(\mathbf{4 a - 4 o})$ displayed two triplets in the range $\delta 2.98-3.46$ and $\delta 3.21-$ 3.61 attributable to $-\mathrm{CH}_{2}$ - protons of piperazine ring and each integrating for four protons except for compounds $4 \mathbf{e}$ 


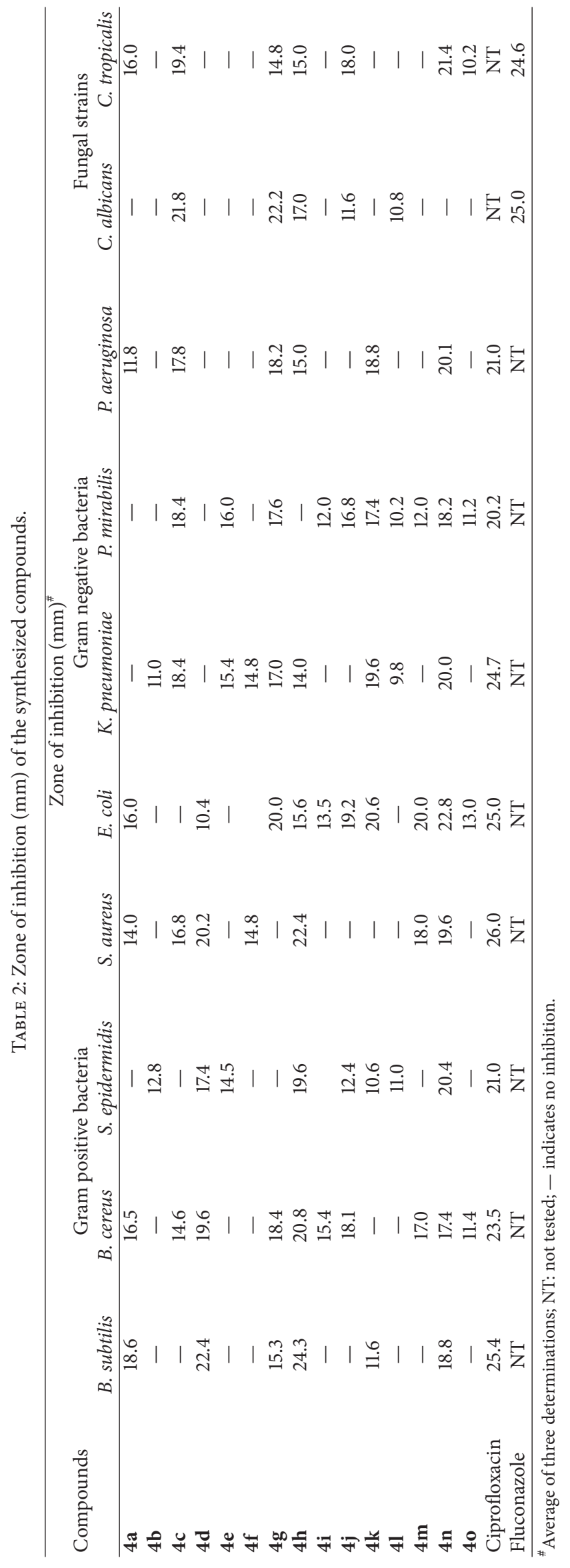




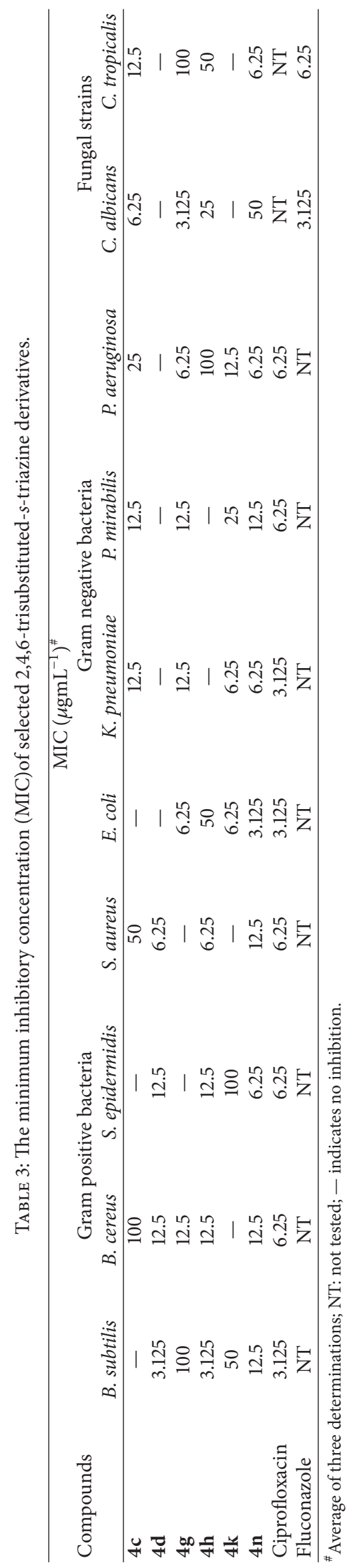


and $4 \mathbf{f}$ that showed multiplet in the range of $\delta 2.75-3.31$. A singlet peak in the range of $\delta 8.31-8.63$ integrating for two protons corresponding to $2^{\circ}-\mathrm{NH}$ linkage was observed for compounds $\mathbf{4 a}, \mathbf{4 c}-\mathbf{4 h}, \mathbf{4 j}$, and $\mathbf{4 m}$ while a singlet in the range $\delta 8.42-8.52$ integrating for one proton corresponding to $-\mathrm{NH}$ attached with $s$-triazine was observed for rest of the compounds. All the other protons belonging toaromatic ring, diethylamino, methyl, and methoxy group were seen accordingly to the expected chemical shift. The ${ }^{13} \mathrm{C}$ NMR depicted the peaks of $s$-triazine nucleus that appeared at the lowest field in the range of $\delta 179.8-183.2 ; \delta 164.6-179.7$; and $\delta 157.5-167.6$ for three nonequivalent carbons. The peaks for two equivalent carbons of piperazine moiety appeared in the range of $\delta 49.4-54.7$ and $\delta 44.6-49.8$ ppm for all the compounds except $4 \mathbf{e}$ and $4 \mathbf{f}$. All other ${ }^{13} \mathrm{C}$ NMR spectra were seen according to the expected chemical shift. The elemental analysis results were within $\pm 0.4 \%$ of thetheoretical values.

3.2. Antimicrobial Activity. The newly synthesized compounds (4a-4o) were evaluated for their antibacterial and antifungal activity against various Gram positive, Gram negative bacteria, and two fungal strains using agar disc diffusion method. The antimicrobial evaluation data is represented in Tables 2-3. The zone of inhibition of all the fifteen 2,4,6trisubstituted-s-triazine derivatives indicates varying degree of inhibition. It was observed that compound $\mathbf{4 d}$ and $\mathbf{4 h}$ displayed equipotent antibacterial efficacy against $B$. subtilis (MIC $3.125 \mu \mathrm{gmL}^{-1}$ ) and S. aureus (MIC $6.25 \mu \mathrm{gmL}^{-1}$ ) under similar experimental conditions with reference to the standard drug. Considering the compounds $\mathbf{4 d}$ and $\mathbf{4 h}$ that bear $N$-methylpiperazine and $N$-(4-nitrophenyl) piperazine moiety, respectively, the compounds also possess a substituted phenyl ring at N4 position of $s$-triazine nucleus. Therefore, it may be suggested that irrespective of the bulkiness of piperazine ring, it is the electron withdrawing group at paraposition of N4 phenyl ring that imparts the activity against Gram positive organism. This is further substantiated by the fact that the compounds substituted with electron releasing groups at para-position of phenyl ring (4a, 4c, 4e, 4f, $\mathbf{4 g}, \mathbf{4 j}$, and $4 \mathrm{~m})$ and compounds with morpholinyl (4b, 4i, 4l, and 4o) and dimethylamino side chain (4k and $4 \mathbf{n}$ ) have showed either no inhibition or minimal inhibition against Gram positive organism. Alternatively, few of the aryl piperazine substituted $s$-triazine $(\mathbf{4 g}, \mathbf{4 k}$ and $\mathbf{4 n})$ showed moderate to good potency against Gram negative bacteria. In particular, the para-fluorophenylpiperazine substituted $s$-triazine (4n) was found to be the most active and exhibited broad spectrum antibacterial activity. This compound was active against $S$. epidermidis, K. Pneumoniae, and P. aeruginosa with an MIC of $6.25 \mu \mathrm{gmL}^{-1}$ whereas for E. coli it showed an MIC of $3.125 \mu \mathrm{gmL}^{-1}$ equipotent with reference to the standard drug. The compound $\mathbf{4 g}$ was found to be active against E. coli and $P$. aeruginosa while compound $4 \mathbf{k}$, wherein the parafluorophenylpiperazine of $\mathbf{4 n}$ has been replaced by parachlorophenylpiperazine, displayed specific activity against Gram negative strain such as E. coli and K. Pneumoniae.

Most of the compounds were incapable of showing effectiveness against the fungal strains under study. However, few compounds (4c, $\mathbf{4 g}$, and $\mathbf{4 n}$ ) have demonstrated antifungal potency comparable to standard fluconazole. Replacement of diethylamino group (4n) with $N 2$ para-methoxyphenyl ring (4c and $\mathbf{4 g}$ ) makes the compounds more active against C. albicans and $\mathbf{4 n}$ was active against $C$. tropicalis that showed equipotent antifungal activity (MIC $6.25 \mu \mathrm{gmL}^{-1}$ ) with reference to fluconazole. Nevertheless, it was not possible to substantially correlate between the structures and antifungal activity as other compounds having phenyl ring substituted with electron releasing group were unable to elicit favourable activity (4a, $\mathbf{4 e}, \mathbf{4 f}, \mathbf{4 j}$, and $\mathbf{4 m})$. Among all the compounds under investigation, compound $\mathbf{4 g}$ demonstrated most significant antifungal activity against $C$. albicans (MIC $3.125 \mu \mathrm{gmL}^{-1}$, zone of inhibition $22.2 \mathrm{~mm}$ ).

\section{Conclusion}

In summary, various 2,4,6-trisubstituted-s-triazine derivatives with varying degree of substitution were synthesized and evaluated for their in vitro antimicrobial activity. The simplicity of their preparation and better yield potentially make $s$-triazines useful and attractive antimicrobial agents. All the compounds were invariably substituted with piperazine, $\mathrm{N}$ methylpiperazine or aryl substituted piperazine as one of the substituents of $s$-triazine scaffold. The tested compounds, $\mathbf{4 d}, \mathbf{4 g}, \mathbf{4 h}, \mathbf{4 k}$, and $\mathbf{4 n}$, have shown considerable in vitro antibacterial efficacy at the range of 3.125-6.25 $\mathrm{gmL}^{-1}$ with reference to the standard drug ciprofloxacin. Irrespective of the bulkiness of piperazine ring, it is the electron withdrawing group at para-position of N4 phenyl ring that imparts good activity against Gram positive organism. From the present study, it may be concluded that the parafluorophenylpiperazine derivatives of $s$-triazine $(4 \mathbf{n})$ were found to be the most active and exhibited broad spectrum antibacterial activity. Among all the compounds under investigation, compound $\mathbf{4 g}$ demonstrated most significant antifungal activity $\left(3.125 \mu \mathrm{gmL}^{-1}\right)$ against $C$. albicans.

\section{Conflict of Interests}

There is no conflict of interests.

\section{Acknowledgments}

The authors are grateful to Banaras Hindu University, Varanasi, India, for providing spectral data of synthesized compounds, and Institute of Pharmacy, HCPG College, Varanasi, for providing the basic facilities.

\section{References}

[1] L. Cantas, S. Q. A. Shah, L. M. Cavaco et al., "A brief multidisciplinary review on antimicrobial resistance in medicine and its linkage to the global environmental microbiota," Frontiers in Microbiology, vol. 4, article 96, 2013.

[2] B. M. Kuehn, "IDSA: better, faster diagnostics for infectious diseases needed to curb overtreatment, antibiotic resistance," 
Journal of the American Medical Association, vol. 310, no. 22, pp. 2385-2386, 2013.

[3] M. Bassetti and E. Righi, "Multidrug-resistant bacteria: what is the threat?" Hematology/the Education Program of the American Society of Hematology, vol. 2013, pp. 428-432, 2013.

[4] R. Zhang, K. Eggleston, V. Rotimi, and R. J. Zeckhauser, "Antibiotic resistance as a global threat: evidence from China, Kuwait and the United States," Globalization and Health, vol. 2, article 6, 2006.

[5] World Health Organization, http://www.who.int/world-healthday/2011/en/index.html.

[6] H. Kothayer, A. A. Elshanawani, M. E. Abu Kull et al., "Design, synthesis and in vitro anticancer evaluation of 4,6diamino-1,3,5-triazine-2-carbohydrazides and -carboxamides," Bioorganic \& Medicinal Chemistry Letters, vol. 23, no. 24, pp. 6886-6889, 2013.

[7] V. Lozano, L. Aguado, B. Hoorelbeke et al., "Targeting HIV entry through interaction with envelope glycoprotein 120 (gp120): synthesis and antiviral evaluation of 1,3,5-triazines with aromatic amino acids," Journal of Medicinal Chemistry, vol. 54, no. 15, pp. 5335-5348, 2011.

[8] H. R. Bhat, S. K. Ghosh, A. Prakash, K. Gogoi, and U. P. Singh, "In vitro antimalarial activity and molecular docking analysis of 4-aminoquinoline-clubbed 1,3,5-triazine derivatives," Letters in Applied Microbiology, vol. 54, no. 5, pp. 483-486, 2013.

[9] N. Sunduru, L. Gupta, V. Chaturvedi, R. Dwivedi, S. Sinha, and P. M. S. Chauhan, "Discovery of new 1,3,5-triazine scaffolds with potent activityagainst Mycobacterium tuberculosis H37Rv," European Journal of Medicinal Chemistry, vol. 45, no. 8, pp. 3335-3345, 2010.

[10] M. Saleh, S. Abbott, V. Perron, C. Lauzon, C. Penney, and B. Zacharie, "Synthesis and antimicrobial activity of 2fluorophenyl-4,6-disubstituted [1,3,5]triazines," Bioorganic \& Medicinal Chemistry Letters, vol. 20, no. 3, pp. 945-949, 2010.

[11] H. R. Bhat, P. K. Pandey, S. K. Ghosh, and U. P. Singh, "Development of 4-aminoquinoline-1,3,5-triazine conjugates as potent antibacterial agent through facile synthetic route," Medicinal Chemistry Research, vol. 22, no. 10, pp. 5056-5065, 2013.

[12] S. N. Gavade, V. L. Markad, K. M. Kodam, M. S. Shingare, and D. V. Mane, "Synthesis and biological evaluation of novel 2,4,6-triazine derivatives as antimicrobial agents," Bioorganic and Medicinal Chemistry Letters, vol. 22, no. 15, pp. 5075-5077, 2012.

[13] U. P. Singh, H. R. Bhat, and P. Gahtori, "Antifungal activity, SAR and physicochemical correlation of some thiazole-1,3,5-triazine derivatives," Journal de Mycologie Medicale, vol. 22, no. 2, pp. 134-141, 2012.

[14] R. B. Singh, N. Das, S. Jana, and A. Das, "Synthesis and in vitro antibacterial screening of some new 2,4,6-trisubstituted-1,3,5triazine derivatives," Letters in Drug Design and Discovery, vol. 9, no. 3, pp. 316-321, 2012.

[15] R. Patel, P. Kumari, and K. Chikhalia, "Novel s-triazinyl piperazines: design, synthesis, characterization and anti-microbial activity," Archives of Applied Science Research, vol. 2, pp. 232240, 2010.

[16] S. Jana and A. Das, "Synthesis and antibacterial activity of some novel 4-benzyl-piperazinyl-s-triazine derivatives," Asian Journal of Chemistry, vol. 25, no. 1, pp. 186-190, 2013.
[17] P. Chaudhary, R. Kumar, A. K. Verma et al., "Synthesis and antimicrobial activity of $N$-alkyl and $N$-aryl piperazine derivatives," Bioorganic and Medicinal Chemistry, vol. 14, no. 6, pp. 1819-1826, 2006.

[18] K. K. Singh, S. C. Joshi, and C. S. Mathela, "Synthesis and in vitro antibacterial activity of $\mathrm{N}$-alkyl and $\mathrm{N}$-aryl piperazine Derivatives," Indian Journal of Chemistry Section B: Organic and Medicinal Chemistry, vol. 50, no. 2, pp. 196-200, 2011.

[19] U. Kulandaivelu, B. Shireesha, C. Mahesh et al., "Synthesis antimicrobial and anticancer activity of $N^{\prime}$ - arylmethylidenepiperazine-1-carbothiohydrazide," Medicinal Chemistry Research, vol. 22, no. 6, pp. 2802-2808, 2013.

[20] C. A. M. Afonso, N. M. T. Lourenço, and A. A. de Rosatella, "Synthesis of 2,4,6-tri-substituted-1,3,5-triazines," Molecules, vol. 11, no. 1, pp. 81-102, 2006.

[21] G. Blotny, "Recent applications of 2,4,6-trichloro-1,3,5-triazine and its derivatives in organic synthesis," Tetrahedron, vol. 62, no. 41, pp. 9507-9522, 2006.

[22] National Committee for Clinical Laboratory Standards, Standard Methods for Dilution Antimicrobial Susceptibility Tests for Bacteria, Which Grow Aerobically, National Committee for Clinical Laboratory Standards, Villanova, Pa, USA, 1982. 

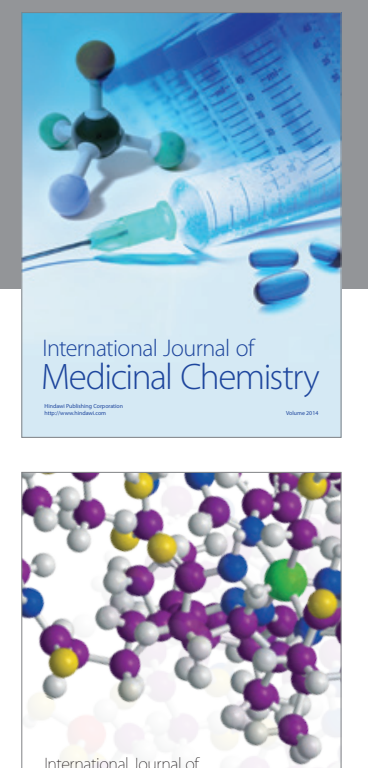

\section{Carbohydrate} Chemistry

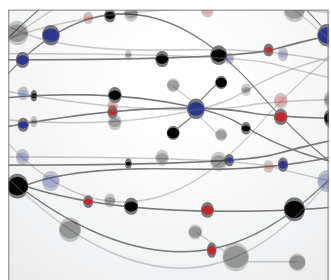

The Scientific World Journal
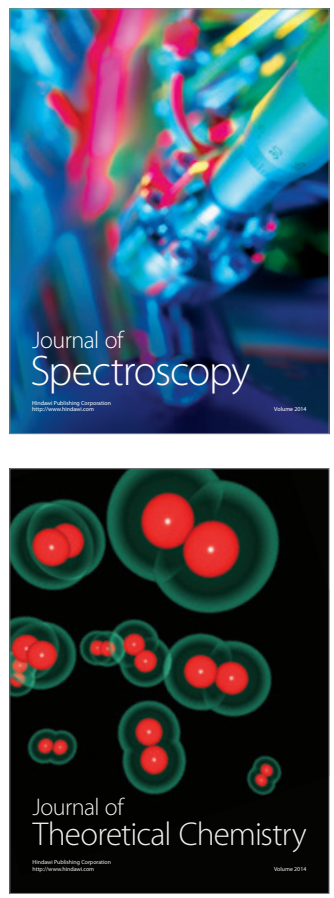
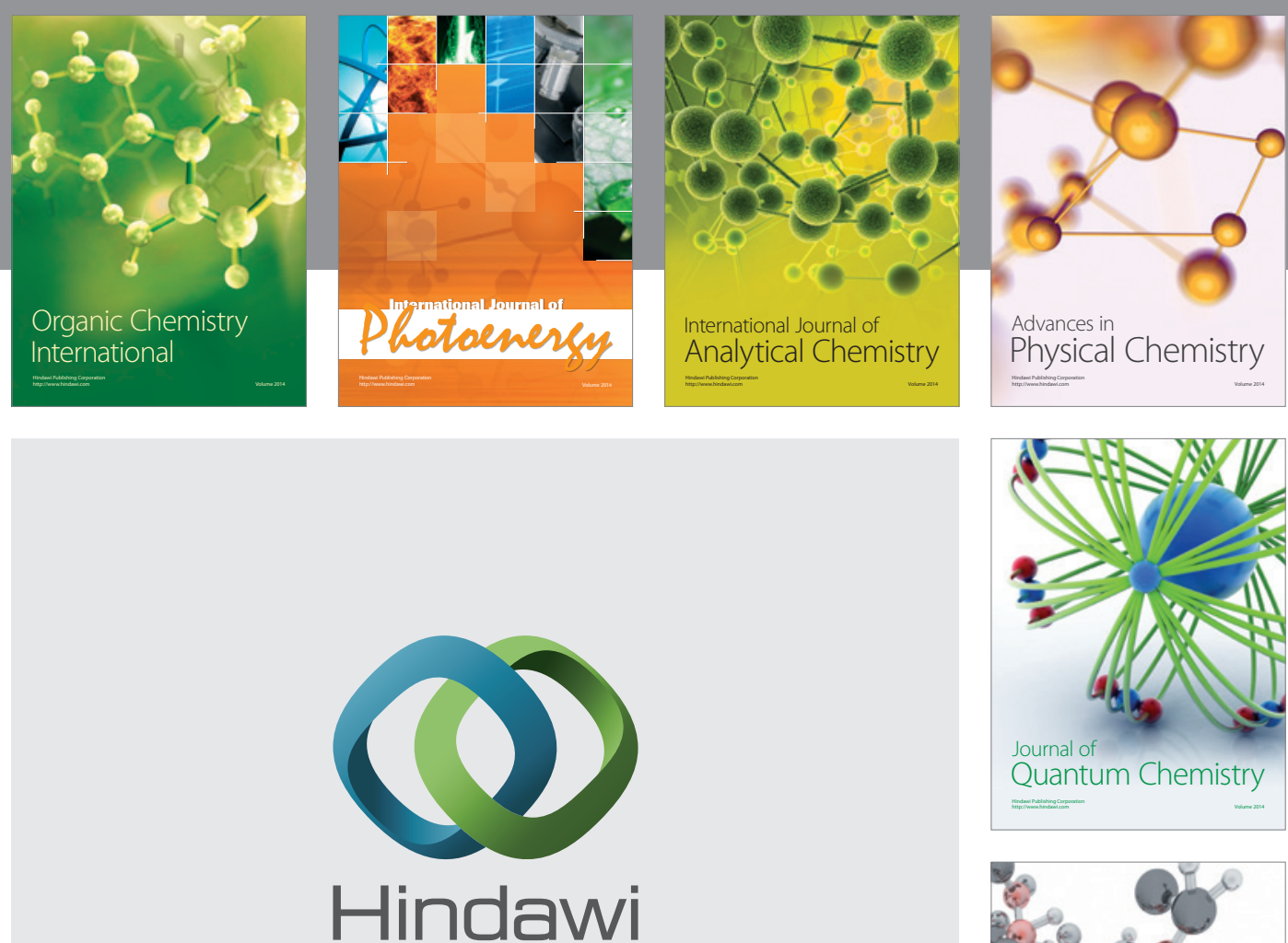

Submit your manuscripts at

http://www.hindawi.com

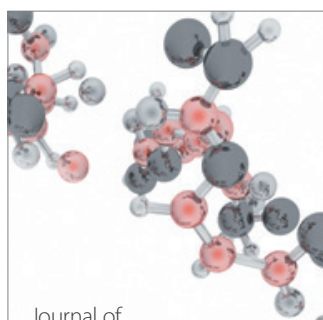

Analytical Methods

in Chemistry

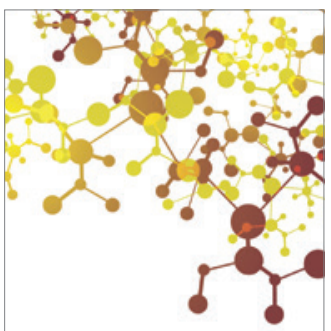

Journal of

Applied Chemistry

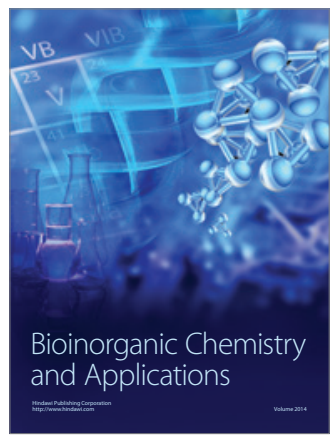

Inorganic Chemistry
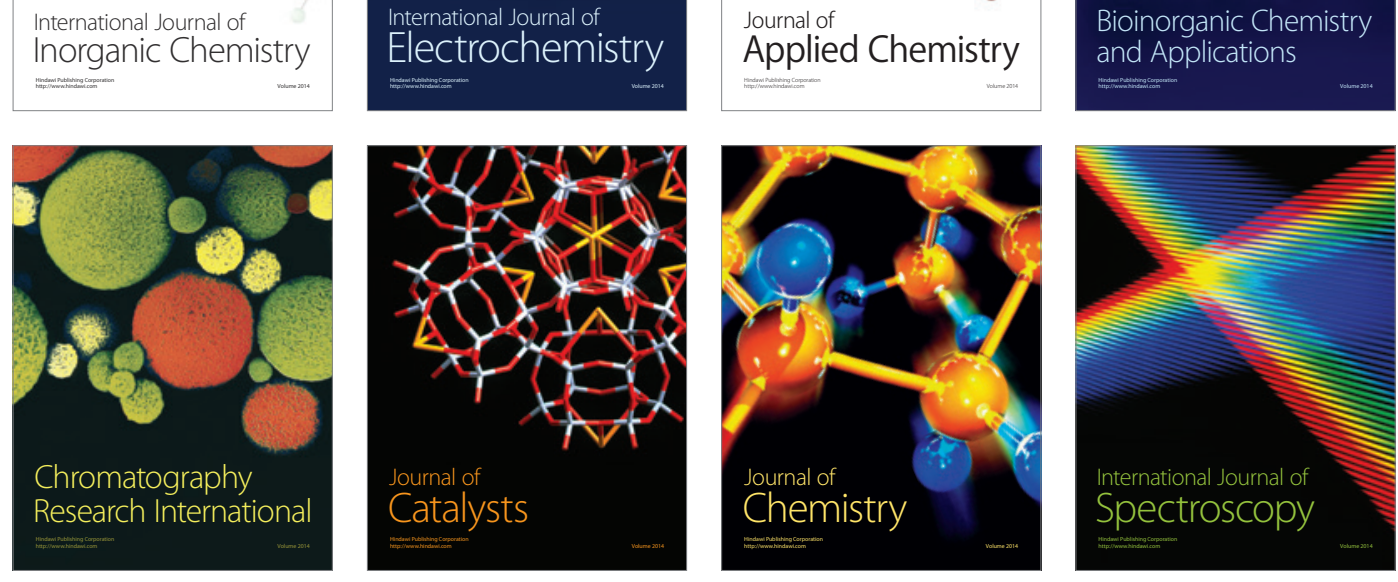